\title{
An Unusual Case Report of Two Bone Osteomyelitis With Long-Term Follow-up
}

\author{
Brian Carpenter, DPM, FACFAS ${ }^{1} \square$, Jeffrey Taylor, DPM ${ }^{2} \rrbracket$, Travis Motley, DPM, FACFAS ${ }^{3}$, \\ Jason Smith, DPM⿻
}

The Foot \& Ankle Journal 1 (4): 1

A 49 year-old patient, with a history of rheumatoid arthritis, presents with suspected septic arthritis of the ankle and subtalar joints. The patient was found to have osteomyelitis of both the talus and calcaneus. The patient was treated by debridement, antibiotic impregnated PMMA beads, and ultimately, a subtalar joint fusion. This case illustrates the importance of the early recognition and diagnosis, the possible sequelae, and the need for aggressive treatment of a septic joint.

Key words: Osteomyelitis, rheumatoid arthritis, subtalar joint, PMMA beads

Accepted: March 1, $2008 \quad$ Published: April 1, 2008

This is an Open Access article distributed under the terms of the Creative Commons Attribution License. It permits unrestricted use, distribution, and reproduction in any medium, provided the original work is properly cited. @The Foot \& Ankle Journal (www.faoj.org)

$\mathbf{O}_{\mathrm{s}}$ steomyelitis is traditionally subdivided into three categories based on etiology. The first type is hematogenous osteomyelitis. This form is commonly monomicrobial, with staphylococcal organisms isolated most frequently. ${ }^{7}$ The second type is direct extension osteomyelitis. It is usually polymicrobial in origin and seen more frequently in the general population. ${ }^{6}$ Sources of direct extension osteomyelitis include puncture wounds, surgery, implants, contiguous ulcerations, and septic arthritis.

\footnotetext{
${ }^{1}$ Director of Residency Training, Associate Professor, University of North Texas Health Science Center, John Peter Smith Hospital, Department of Orthopaedics, 1500 South Main Street, Ft.Worth, Texas 76104.

${ }^{2}$ Private Practice; North Texas Podiatry, 401 Westpark Way, Euless.

Texas

${ }^{3}$ Staff Physician, Assistant Professor, University of North Texas Health Science Center, Department of Orthopaedics. 1500 South Main Street, Ft. Worth, Texas 76104.

${ }^{4}$ Resident, PGY-2,John Peter Smith Hospital, Department of Orthopaedics. 1500 South Main Street, Ft. Worth, Texas 76104

The third type is osteomyelitis secondary to vascular insufficiency. It is also polymicrobial in origin and seen mostly in those over 55 years of age as the result of tissue hypoxia. ${ }^{1}$

Septic arthritis is the most destructive form of arthritis. It is classified by its offending pathogen or etiology. Neisseria gonorrbea is commonly isolated from sexually active adults with associated superficial lesions. ${ }^{8}$ Haemophilus influenza is commonly isolated from children under the age of two. ${ }^{8} \quad$ Staphylococcus and Streptococcus species, which are the most common offending pathogens overall, are seen in children over the age of two and in adults. ${ }^{8}$ The etiologies are very similar to osteomyelitis.

(C) The Foot \& Ankle Journal, 2008 


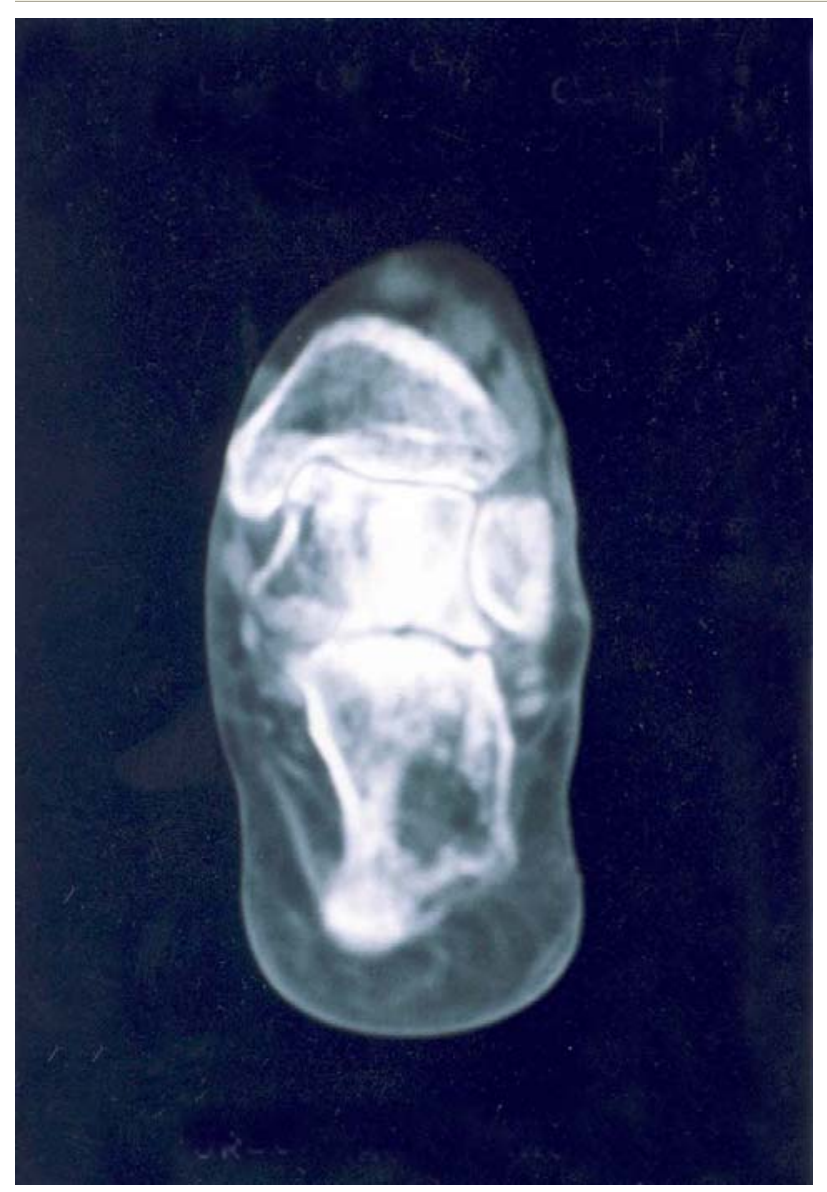

Figure 1 CT scan revealed joint narrowing consistent with degenerative arthritis. A large area of bone lysis is seen in the body of the os calcis.

Hematogenous septic arthritis may be secondary to upper respiratory and skin infections, most frequently encountered with gram-positive organisms. ${ }^{8}$

A joint is susceptible to infection because of the profound vascular supply within the synovial joint lining. The joint may be even more prone to an infective process given an existing arthritide, such as rheumatoid arthritis.

Arthritis not only causes direct damage to the joint, but may also be treated with repeated corticosteroid injections that can decrease the joint's immunocompetence.
Direct extension septic arthritis shares its etiologies with direct extension osteomyelitis, but septic arthritis may also originate from an adjacent osteomyelitis. $^{1}$

The following case report describes a middle-aged female with a history of rheumatoid arthritis, who developed osteomyelitis of the talus and calcaneus. The joints were never proven to be septic by joint aspiration and culture. The ensuing destructive sequelae raises many questions about the origin and the development of the infective process. ${ }^{1}$

\section{Case Report}

A 49 year-old white female with a past medical history of long term rheumatoid arthritis and a sedentary lifestyle, presented to the clinic complaining of a painful left ankle. The ankle became painful, erythematous, and edematous three months prior, and was accompanied by fever and chills. The patient did not initially seek treatment. Ten to fourteen days later, with rest and a decrease in activity, the erythema and edema subsided without the use of antibiotics, however, the pain persisted. The patient then went to see her rheumatologist, who treated her with physical therapy, an NSAID, and a steroid injection of the left ankle. The patient denied trauma, but did note a superficial cat scratch to the anterior aspect of her left lower leg one to two weeks prior to the onset of pain, which she treated with a topical antibiotic.

On initial physical examination significant findings revealed a globally edematous left ankle. The swelling was markedly more edematous on the lateral aspect of the ankle. There was pain on palpation of the anterior, lateral, and posterior aspect of the ankle joint. Pain was elicited with eversion and dorsiflexion of the ankle and there was no pain of the subtalar joint with range of motion. The neurovascular status of both extremities was intact. 


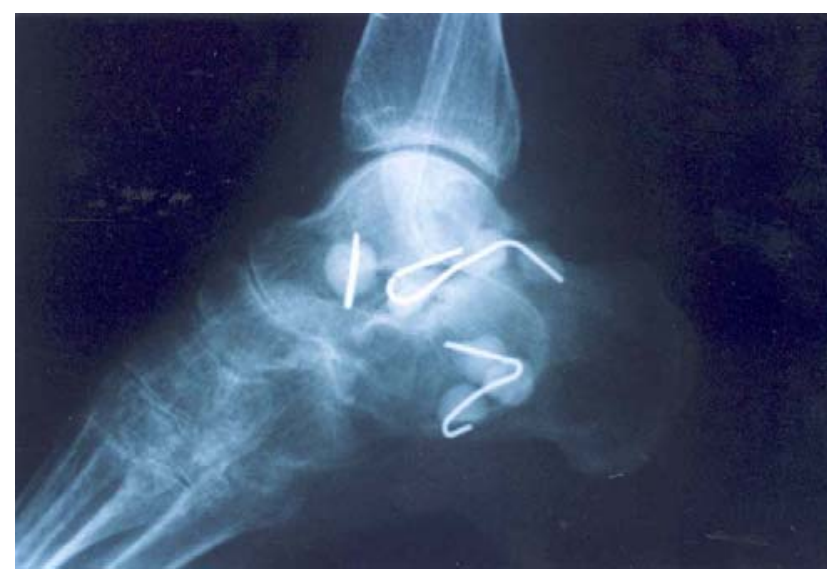

Figure 2 Following surgical debridement of osteomyelitic bone, antibiotic impregnated PMMA beads were inserted. Cultures revealed staphylococcus aureus osteomyelitis.

Plain films of the left foot and ankle, taken on a previous presentation did not reveal a fracture or dislocation, although there was significant degenerative joint disease of the left ankle joint.

The patient returned to the clinic two weeks later with an additional complaint of a painful left heel on weightbearing. Physical exam revealed severe pain with compression to the body of the left calcaneus. There was no apparent cellulitis of the left foot or ankle. Computerized tomography (CT) scan findings of the left foot and ankle revealed degenerative joint disease of the subtalar joint with an irregular calcaneal-cuboid joint that includes significant destruction of bone. A large lytic lesion in the posterior inferior aspect of the calcaneus is seen with a small lytic lesion of the medial talar dome. There were trabecular changes of the lateral talar body with surrounding soft tissue inflammation. (Fig. 1)

Plain films, taken shortly after that time, corresponded with the CT findings. We concluded that degenerative changes of the talus, calcaneus, and subtalar joint were probably the result of an infective process.

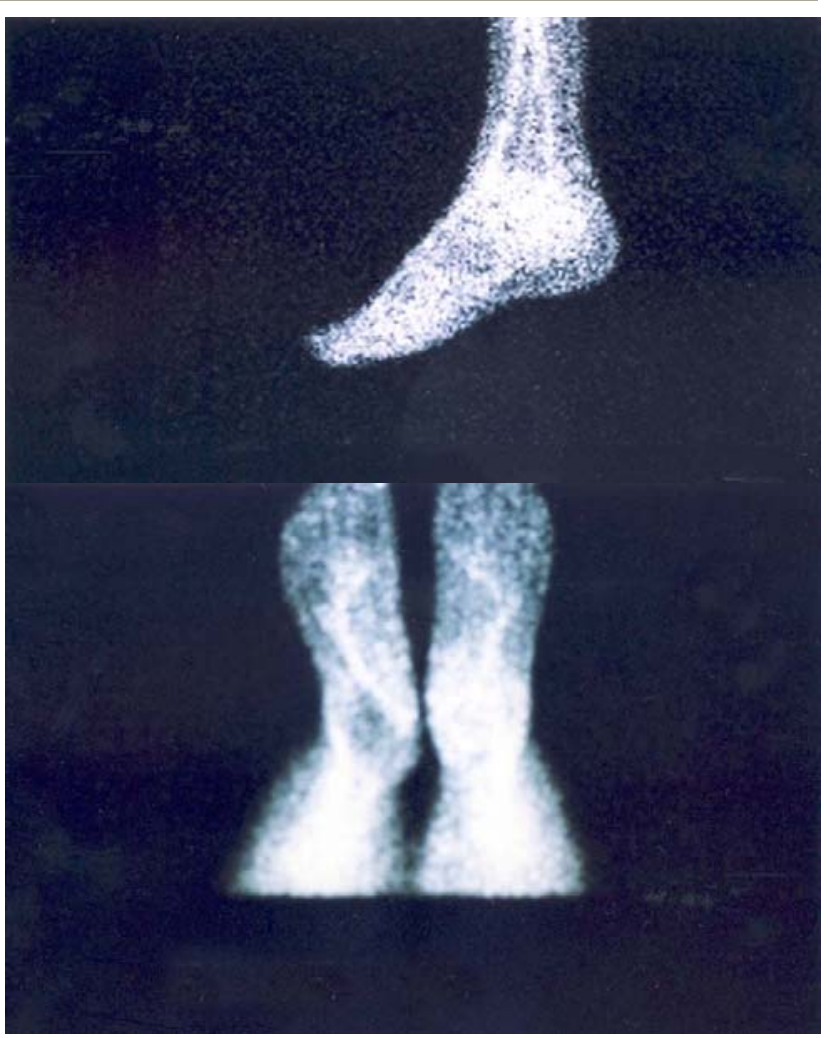

Figure 3 Ceretec white blood cell bone scans performed six weeks after insertion of PMMA beads were negative for any signs of active osteomyelitis.

Five days later, surgical debridement of osteomyelitic bone of the left talus and calcaneus was performed to an intra-operative viable periphery. Tobramycin impregnated beads were inserted into the dead space, and the patient was placed on cephazolin 2 gm IV every 8 hours. (Fig. 2)

The wound was closed over the implanted beads with a drain in place. Bone biopsy of debrided bone was positive for osteomyelitis, and biopsy of peripheral bone and residual bone was negative. Cultures of the debrided necrotic bone were positive for Staphylococcus aureus. The postoperative period was unremarkable. A Ceretec WBC-labeled bone scan performed six weeks after the initial debridement. This was negative for osteomyelitis (Fig. 3) 


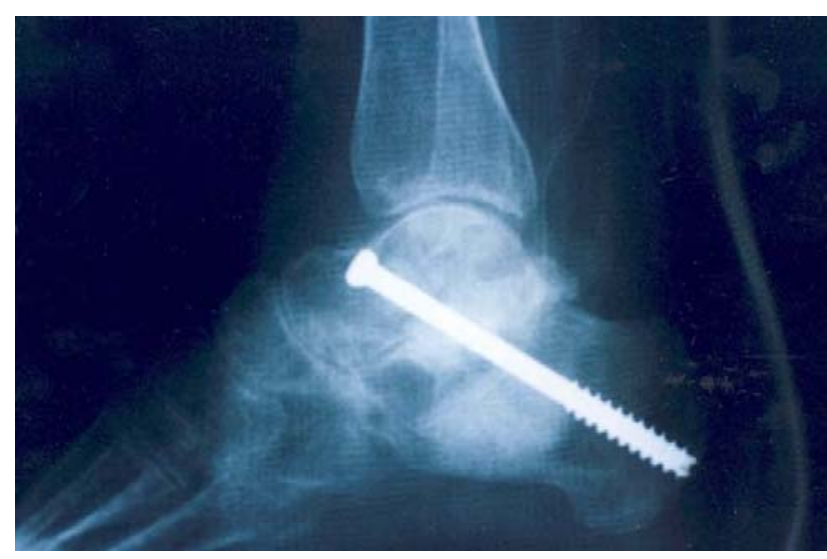

Figure 4 Iliac bone graft is used with a single cannulated cancellous screw to fuse the subtalar joint.

Ten weeks after debridement of the left talus and calcaneus, the beads were removed. The residual bone of the talus and calcaneus were clinically viable, and a subtalar joint fusion with talar and calcaneal reconstruction using autogenic iliac bone graft was performed. The patient was placed in a below-the-knee cast. Plain film findings two weeks after STJ fusion demonstrated intact internal fixation from anterior dorsal to plantar posterior with good placement and joint alignment. A portion of increased density of both the talus and calcaneus corresponded to generalized osteopenia of the bone grafts. (Fig. 4) Intravenous antibiotics were discontinued and the patient was put on a two-week course of oral cephalexin.

The postoperative course involved a nonweightbearing, below-the-knee cast for eight weeks with progression to a non-weightbearing removable cast walker (RCW) for three weeks. The patient was then placed in a weightbearing RCW for another three weeks. Proper foot orthoses and ankle brace was fitted when the patient progressed to her shoes.

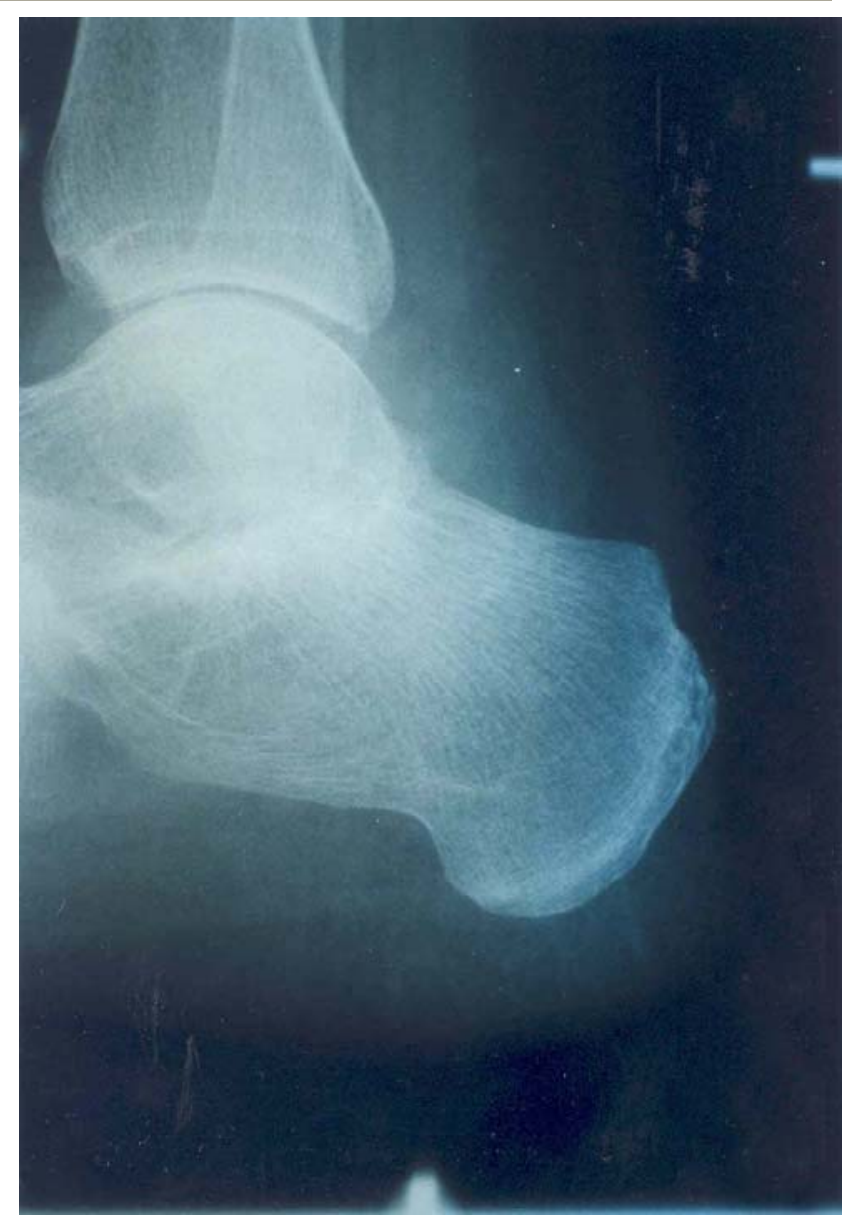

Figure 5 Six months after fusion, the subtalar joint is stable and without pain on ankle and foot range of motion.

After casting, the patient underwent eight weeks of physical therapy to increase range of motion, proprioception, and strength. The patient was pain free six months after fusion. The plain films demonstrated approximately $90 \%$ fusion of the left subtalar joint with the heel in rectus position. (Fig. 5)

The patient is now ten years post surgery. She is enjoying a healthy lifestyle that includes jogging without pain. 


\section{Discussion}

There are many questions that arise from this case report and should be addressed to make any pertinent conclusions.

The patient's history suggests clinical sepsis prior to presenting and the etiology remains unclear. First of all, the cat scratch she reported was probably not the cause. We were unable to isolate the most common organism isolated from cat scratches or bites, Pasteurella multiocida. ${ }^{2}$ Also, the location and depth of the laceration are not consistent with an infectious introduction of the ankle or subtalar joint. Secondly, a steroid injection, which has been demonstrated as a source of joint sepsis in the literature, was administered to the patient early in the presentation. Injections of corticosteroid into a septic joint may increase the propagation of the infectious process, but cannot be labeled as the cause due to the onset of symptoms prior to the injection. ${ }^{3,4}$ Finally, the patient's long term rheumatoid arthritis must be recognized as a significant factor as to increased risk of joint sepsis, but cannot be labeled as a cause. ${ }^{5,6}$ Given the exclusion of these three factors and the absence of other complicating trauma, although rarely seen in middle aged adults, we hypothesize the septic arthritis must be attributed to a hematogenous route. ${ }^{5,6}$

Assuming bacteremia one must decide on the location of the infection. Given the patient's report, it is easy to assume ankle joint sepsis. The suspicion is heightened by the possible propagating of infection by a local joint injection to the ankle joint. The clinical and radiographic findings also suggest extra-tibiotalar sepsis. An effusion of the subtalar joint may present with ankle joint swelling that is more prominent below the lateral malleolus, given the communicating lateral anatomy of the subtalar and ankle joints. Computerized tomography of the foot and ankle further supports the hypothesis of subtalar sepsis by displaying adjacent boney lesions located in the inferior talus and superior calcaneus.
Therefore, the original septic joint may have been the subtalar joint in isolation or in combination with the ankle joint. It appears the detrimental sequelae of osteomyelitis developed only in the subtalar joint.

Sequelae of septic arthritis may include persistence of the infection secondary to inadequate drainage. It can also be caused by damage to the articular cartilage, tenosynovitis, and secondary osteomyelitis. As intra-articular exudate increases, the joint attempts to accommodate its increased volume through posture, which causes more pain and stiffness. When the joint can no longer handle the intraarticular pressure, the corresponding cartilage is compressed, synovial blood supply is compromised, capsular erosions and sinus tracts form, and contiguous spread to underlying bone results. ${ }^{5}$

When osteomyelitis is recognized secondary to joint sepsis, joint resection arthroplasty, staged arthrodesis, or even amputation can be performed to alleviate the patient's symptoms and stop further spread of the infection. ${ }^{5} \quad$ Surgical debridement of all necrotic bone must be performed. In this case, it was followed by implantation of PMMA antibiotic beads for the treatment of existing infection and prophylaxis of further infection in the anatomical dead space. The use of antibiotic laden beads is efficacious in the treatment of acute and chronic osteomyelitis. PMMA beads have been in use for over 30 years. The beads are typically left in the wound for 5 to 14 days after debridement or until soft tissure coverage or primary closure can be achieved. ${ }^{13}$ Studies have shown that minimum inhibitory concentration of antibiotics are released from PMMA beads from the first 2 days after implantation up to multiple weeks. ${ }^{12}$ Following proper debridement and antibiotic prophylaxis, a Ceretec bone scan, which is a sensitive and specific for osteomyelitis, can be 
utilized to confirm the absence of infection or persistent infection. An isolated talocalcaneal arthrodesis, employing an iliac crest bone graft, has been described by many authors as an excellent way to treat various degenerative processes of the subtalar joint, and has proven to be a more functional alternative to a pan-talar fusion. ${ }^{9,10}$

This patient most likely developed subtalar septic arthritis from an uncommon hematogenous route. The infection was not drained and may have been even propagated with a corticosteroid injection and spread contiguously to the talus and calcaneus. Once recognized, the osteomyelitis was aggressively treated with surgery and appropriate intravenous antibiotic therapy. The case illustrates three important points about the septic joint. First, the suspicion of a septic joint must be diagnosed and treated appropriately and aggressively by joint aspiration, if possible, and proper cultures and definitive antibiotics. Secondly, early intervention in these cases is paramount, as demonstrated graphically by this case. Finally, if complications, such as osteomyelitis, do occur, proper aggressive surgical treatment must be performed promptly.

\section{References}

1. Joseph, W. : Infections, in Principles and Practice of Podiatric Medicine, Levy, Hetherington (eds.),

2. New York, Churchill Livingstone, 1990, 275-313.

3. Chodakewitz, J., Bia, F. : Septic Arthritis and Osteomyelitis form a Cat Bite. Yale Journal of Biology and Medicine, 61:513-518, 1988.

4. Kothari, T. et al. : Pseudomonas cepacia Septic Arthritis due to Intra-articular Injections of Methylprednisolone. Canadian Medical Association Journal. 116(11): 1230, 1232, 1235, June 1977.

5. Gowans, J., Granieri, P. : Septic Arthritis. Its Relation to Intra-articular Injections of Hydrocortisone Acetate. New England Journal of Medicine 261: 502-503, 1959.

6. Freeland, A., Senter, B. : Septic Arthritis and Osteomyelitis. Hand Clinics 5: 533-552, November 1989.

7. Cunningham, R., Cockayne, A., Humphreys, H. : Clinical and Molecular Aspects of the Pathogenesis of Staphylococcus aureus and Bone and Joint Infections. Journal of Medical Microbiology 44(3): 157-164, March 1996.

8. Sternbach, G., Baker, F. : The Emergency joint: Arthrocentesis and Synovial Fluid Analysis. JACEP 5: 787792, October 1976.

9. Calhoun, J., Mader, J. : Antibiotic Beads in the Management of Surgical Infections. American Journal of Surgery 157: 443-449, April 1989.

10. Russotti, G. et al. : Isolated Talocalcaneal Arthrodesis. Journal of Bone and Joint Surgery 79-A: 1472-1478, December 1988.

11. Thomas, F. : Arthrodesis of the Subtalar Joint. Journal of Bone and Joint Surgery 49-B: 93-97, February 1967.

12. Perry, A. et al : Antimicrobial Realease Kinetics From Polymethylmethacrylate in a Novel Continous Flow Chamber. Clinical Orthopaedics and Related Research 403, pp. 49-53 2002.

13. Walenkamp, G. et al : Osteomyelitis Treated With Gentamicin-PMMA Beads. Acta Orthop Scand 69 (5): 518522, 1998. 